\title{
COMPORTAMENTO VEGETATIVO DE SEIS CULTIVARES DE COQUEIRO- ANÃO (Cocos nucifera L.), EM SANTO ANTÔNIO DE LEVERGER - MT ${ }^{1}$
}

\author{
MARCOS PÓVOAS JUCÁ², HILTON NEY GAÍVA, WALTER ESFRAIN PEREIRA ${ }^{4}$, ADÉLCIO MILESKI $^{5}$
}

\begin{abstract}
RESUMO - A utilização de variedades ou genótipos de coqueiro adaptados às condições edafoclimáticas de uma região é de fundamental importância para a obtenção de altas produtividades. Neste experimento, estudou-se o comportamento vegetativo de seis cultivares de coqueiro-anão no município de Santo Antônio de Leverger - MT. As cultivares avaliadas foram: Anão-Vermelho-daMalásia (AVM), Anão-Verde-de-Jiqui (AVeJ), Anão-Vermelho-de-Gramame (AVG), Anão-Vermelho-de-Camarões (AVC), Anão-Amarelode-Gramame (AAG) e Anão-Amarelo-da-Malásia (AAM) plantadas em maio de 1998, nas quais foram avaliadas, de abril de 1999 a dezembro de 2000, as seguintes características: circunferência do coleto (CC), número de folhas emitidas (NFE), número de folhas vivas (NFV), número de folhas mortas (NFM), número de folíolos na folha 3 (NFoF3), comprimento de folíolo na folha 3 (CFoF3), comprimento da folha 3 (CF3) e comprimento de pecíolo na folha 3 (CPF3). Utilizou-se delineamento experimental em blocos casualizados, com quatro repetições, tendo cada parcela 16 plantas úteis. Para as características circunferência do coleto (CC), número de folíolos na folha 3 (NFoF3) e comprimento da folha 3 (CF3), o AAM apresentou os menores valores. O AVeJ e AVM apresentaram maiores crescimentos de planta, enquanto o AAM, AAG e AVC apresentaram menor crescimento; já o AVG apresentou desempenho intermediário.
\end{abstract}

Termos para indexação: Cocos nucifera L., crescimento, componentes principais

\section{VEGETATIVE BEHAVIOUR OF SIX CULTIVARS OF DWARF COCONUT PALM (Cocos nucifera L.) IN SANTO ANTÔNIO DE LEVERGER - MATO GROSSO STATE - BRAZIL}

\begin{abstract}
The use of cultivars of coconut palm suitable to the edaphoclimatic conditions of a region is of basic importance for the attainment of high productivity. With the objective to study the vegetative behavior of six cultivars of dwarf coconut palm was carried out an experiment in Santo Antônio de Leverger - Mato Grosso State. The cultivars studied were: Anão Vermelho da Malásia (AVM), Anão Verde de Jiqui (AVeJ), Anão Vermelho de Gramame (AVG), Anão Vermelho de Camarões (AVC), Anão Amarelo de Gramame (AAG) and Anão Amarelo da Malásia (AAM), planted in May of 1998, in which were evaluated from April of 1999 till December of 2000 the following characteristics: girth circumference (CC), number of emitted leaves (NFE), number of alive leaves (NFV), number of dead leaves (NFM), number of leaflets in the leaf 3 (NFoF3), length leaflets in the leaf 3 (CFoF3), leaf 3 length (CF3) and petiole length in the leaf 3 (CPF3). It was utilized a randomized block design with four repetitions, having in each plot 16 plants. For the girth circumference (CC), number of leaflets in the leaf 3 (NFoF3) and leaf 3 length (CF3), the AMM presented the lessers values. The AVeJ and AVM were more vigorous when compared to AAM, AAG and AVC.
\end{abstract}

Index terms: Cocos nucifera $\mathrm{L}$. , growth, principal components

\section{INTRODUÇÃO}

O coqueiro é uma cultura predominantemente cultivada por pequenos produtores, com áreas plantadas que variam de 0,5 a 4,0 ha (Persley, 1992). As perspectivas para a cultura são promissoras, pois há mercado potencial muito grande a ser explorado com água de coco. Hoje, a industrialização desta (envazamento) permite o aumento da vida útil do produto, possibilitando, assim, crescimento substancial no consumo (Ribeiro et al., 1999). Em relação à água de coco "in natura", por exemplo, novas alternativas de mercado têm surgido nos últimos anos, contribuindo para aumentar o consumo do produto. Uma delas foi a introdução de máquinas para sua extração, as "Coco Express", com o produto servido diretamente ao consumidor (Saabor et al., 2000).

A produtividade média brasileira ainda é baixa, na ordem de 20 a 30 frutos/planta/ano (Aragão et al., 1997), e no Estado de Mato Grosso não é diferente, pois estão sendo plantandos genótipos não selecionados, como, também, ocorre manejo inadequado da cultura.

Diversos trabalhos realizados, em várias partes do mundo (Akpan, 1994; Zushun, 1994; Bourdeix et al., 1992), evidenciam a

1 (Trabalho 108/2001). Recebido: 25/05/2001. Aceito para publicação: 01/05/2002. Parte da tese do primeiro autor apresentada à Universidade Federal de Mato Grosso, para a obtenção do grau de Mestre em Agricultura Tropical.

2 Engo Agro M. Sc., Faculdade de Agronomia e Medicina Veterinária (FAMEV) da Universidade Federal de Mato Grosso (UFMT). Bolsista da CAPES. Av. Fernando Corrêa da Costa, s/n, Cuiabá-MT, CEP 78068-900.

3 Eng $^{\mathrm{o}}$ Agr $^{\mathrm{o}}$ D. Sc., Professor Adjunto - FAMEV/UFMT.

4 Eng ${ }^{\mathrm{o}} \mathrm{Agr}^{\mathrm{o}} \mathrm{D}$. Sc., Bolsista do CNPq - DCR, FAMEV/UFMT.

5 Estudante de Agronomia da FAMEV, UFMT. 
importância da avaliação do grau de adaptabilidade de variedades ou de genótipos de coqueiro às condições edafoclimáticas de um determinado local. Atualmente, há grande interesse entre os principais países produtores de coco do mundo, na avaliação e seleção de híbridos, para solucionar seus problemas de produção, com pragas, doenças e de adaptação edafoclimática (Nuce de Lamothe et al., 1991). Nas Filipinas, a escolha de variedades melhor adaptadas às condições edafoclimáticas é uma das principais medidas recomendadas para evitar o decréscimo do rendimento observado nos últimos anos (Aldaba, 1995).

O presente estudo teve como objetivo avaliar o comportamento vegetativo de cultivares de coqueiro-anão, nas condições da região de Santo Antônio de Leverger, Estado de Mato Grosso.

\section{MATERIAL E MÉTODOS}

O experimento foi conduzido na Fazenda Experimental da Universidade Federal de Mato Grosso, no município de Santo Antônio de Leverger, Mato Grosso, no período de abril de 1999 a dezembro de 2000. Essa região localiza-se a $15^{\circ} 47^{\prime} 11^{\prime \prime} \mathrm{S}$ e 56 $04^{\circ} 17^{\prime \prime} \mathrm{W}$, com $179 \mathrm{~m}$ de altitude, apresenta o clima do tipo tropical quente e subúmido. O solo da área experimental foi classificado como Latossolo Amarelo, eutrófico câmbico, A moderado, textura argilosa, fase Cerrado, relevo plano. Utilizouse a irrigação por meio de microaspersores, em dois turnos de 3

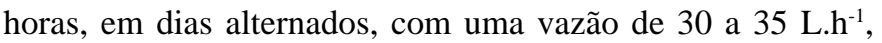
baseado em avaliações realizadas na área experimental. $\mathrm{O}$ pomar recebeu os tratos culturais normalmente recomendados para a cultura (Ferreira et al., 1998). As cultivares utilizadas foram: AnãoVermelho-da-Malásia (AVM), Anão-Verde-de-Jiqui (AVeJ), AnãoAmarelo-da-Malásia (AAM), Anão-Vermelho-de-Camarões (AVC), Anão-Vermelho-de-Gramame (AVG) e Anão-Amarelo-deGramame (AAG), plantadas em espaçamentos de 7,5 m x 7,5 m x 7,5 m em triângulo eqüilátero, no mês de maio de1998 .

A metodologia utilizada na determinação das características selecionadas para este estudo, a cada 28 dias, é descrita a seguir: a) Circunferência do coleto: efetuada a $5 \mathrm{~cm}$ do solo, com auxílio de uma fita métrica.

b) Número de folhas vivas número de folhas verdes e adultas com mais de $50 \%$ da folha aberta.

c) Número de folhas emitidas: número de folhas com abertura superior a $50 \%$, onde se marcava com fita plástica a última folha emitida, para se evitar futura recontagem. Assim, as folhas emitidas entre duas sucessivas avaliações, eram as existentes entre aquela marcada (exclusive) e a última aberta por ocasião da leitura.

d) Número de folhas mortas: contagem de folhas que apresentavam mais de $50 \%$ da área foliar seca.

e) Número de folíolos na folha três: contagem de folíolos da folha 3, ou seja, a terceira folha completamente aberta a partir do ápice para a base da planta.

f) Comprimento da folha três: determinado com auxílio de uma fita métrica.

g) Comprimento do pecíolo da folha três: comprimento entre a inserção da folha três no estipe até o início do folíolo.

h) Comprimento do folíolo na folha três: média de três medidas de folíolos centrais, utilizando-se de uma fita métrica.
O delineamento experimental foi o de blocos ao acaso, com seis tratamentos, quatro repetições e 16 plantas por parcela. Os dados obtidos foram submetidos à análise de variância e ao teste de Tukey, a 5\% de probabilidade. Devido à elevada correlação existente entre as variáveis determinadas, e com o objetivo de facilitar a classificação das cultivares, os valores obtidos foram submetidos à análises de componentes principais (Cruz e Regazzi, 1994; Johnson e Wichern, 1998). O agrupamento das cultivares foi baseado no método de Tocher, aplicado nos escores dos componentes principais. Algumas aplicações dos componentes principais na agricultura foram apresentadas por Iezzoni e Pritts (1991), Caradus et al. (1993) e Stewart et al. (1998).

\section{RESULTADOS E DISCUSSÃO}

A circunferência do coleto de todas as cultivares aumentou ao longo dos meses avaliados. A cultivar AAM apresentou menor desempenho para essa característica (Figura 1). Comparando o valor obtido pelo AVM aos 19 meses após o plantio, que foi de $62 \mathrm{~cm}$, aos $60 \mathrm{~cm}$ verificados em coqueiro híbrido (AVMxGPY) por Ferraz et al. (1987), no Estado de Pernambuco, verificou-se crescimento vegetativo satisfatório das cultivares avaliadas nas condições locais, visto que os híbridos apresentam maior crescimento vegetativo que os anões.

$\mathrm{O}$ número de folhas vivas (NFV), número de folhas emitidas (NFE) e o número de folhas mortas (NFM) de todas as cultivares não diferiram estatisticamente entre si no período da avaliação (Figura 1). Apenas o NFV não é suficiente para conhecer o potencial da planta para produzir fotoassimilados, sendo necessário conhecer também o número de folíolos na folha 3 , o comprimento da folha 3 e o comprimento do folíolo na folha 3 , os quais fornecem melhor estimativa da área foliar da planta, de difícil determinação em condições de campo. No mês de junho/ 00, houve decréscimo dessas características, possivelmente, devido ao déficit hídrico, uma vez que não houve precipitação pluviométrica nesse período, e o sistema de irrigação só foi instalado ao final do mesmo, e verificou-se aumento dessas características nas avaliações posteriores. O maior número de folhas vivas aumenta a área fotossintética, ocorrendo maior disponibilidade de fotoassimilados para o crescimento da planta. Quando cultivado sob condições desfavoráveis, como seca ou deficiência nutricional, ocorre redução no número de folhas vivas por planta, devido à diminuição do ritmo de emissão foliar e não pela morte precoce das folhas (Passos, 1998).

Comparando os valores médios obtidos pelo AVeJ, aos 24 meses após o plantio, para o número de folhas vivas e número de folhas mortas que foram de 11 e 1 , respectivamente, às nove folhas vivas e uma e meia morta verificados em coqueiro híbrido (AVeJxGBrRN) por Aragão et al. (1997), no Estado de Sergipe, percebe-se um indicativo da aptidão da região para a cocoicultura. Comparando o valor obtido pelo AVM aos 24 meses após o plantio, que foi de uma folha emitida, a uma folha emitida verificado em coqueiro híbrido (AVMxGOA) por Santos et al. (1982).

O número de folíolos na folha 3 (NFoF3), comprimento da folha 3 (CF3) e comprimento dos folíolos na folha $3(\mathrm{CFoF} 3) \mathrm{de}$ todas as cultivares avaliadas aumentaram ao longo do período da avaliação (Figura 2). O AAM apresentou menor desempenho

Rev. Bras. Frutic., Jaboticabal - SP, v. 24, n. 2, p. 463-467, agosto 2002 
para as características de número de folíolos na folha 3 e comprimento da folha 3. Para comprimento dos folíolos na folha 3, só houve diferença significativa na avaliação de julho/99. O AAG apresentou menor desempenho nos meses de setembro/99, junho/00, julho/00, agosto/00 e outubro/00 para a característica de comprimento da folha 3 . O número de folíolos na folha 3, com 133 $\mathrm{cm}$, comprimento da folha 3, com $193 \mathrm{~cm}$ e comprimento dos folíolos, com $87 \mathrm{~cm}$ para o AVeJ, aos 25 meses, quando comparados ao AVeJ estudados por Ribeiro (2000), com valores máximos de $144 \mathrm{~cm}, 120 \mathrm{~cm}$ e $43 \mathrm{~cm}$, respectivamente, indicam desempenhos adequados dessas características para as condições da área estudada.

O comprimento do pecíolo na folha 3 (CPF3) apresentou maiores valores na AVG, nos meses de setembro/99, março/00, julho/00 e outubro/00, e o AVeJ nos meses de junho/00, julho/00, agosto/00 e setembro1/00. O comprimento do pecíolo na folha 3 , associado aos menores comprimentos da folha 3 e do número de folíolos, verificados nas cultivares anãs, são características importantes no sentido de se permitir melhor arranjo na densidade de plantio e devendo ser largo e curto a fim de suportar o próprio peso da folha, além do peso dos cachos. Comparando o valor obtido pelo AveJ, aos 25 meses após o plantio, que foi de $27 \mathrm{~cm}$, aos $23 \mathrm{~cm}$ verificados ao AVeJ por Ribeiro (2000), verifica-se desempenho adequado dessa característica, para as condições da área estudada.

A análise de componentes principais, aplicada na matriz de correlações, permitiu identificar dois componentes principais que foram significativos (Tabela 1), os quais explicam $71,1 \%$ da variância total.

O primeiro componente principal basicamente contrasta as características relacionadas com o crescimento da planta e o número de folhas mortas. O segundo componente principal representa o número de folhas emitidas e o comprimento do pecíolo.

A classificação das cultivares avaliadas pode ser observada na Figura 3, detectando-se três grupos: o primeiro grupo formado por AVeJ e AVM, o qual apresentou maior crescimento e menor número de folhas mortas; o segundo grupo formado por AAM, AAG, AVC, o qual apresentou o menor crescimento e maior número de folhas mortas e o terceiro grupo formado por AVG de comportamento intermediário. Bourdeix et al. (1992) utilizaram, também, os componentes principais para a classificação dos genótipos utilizados por eles, identificando quatro grupos.
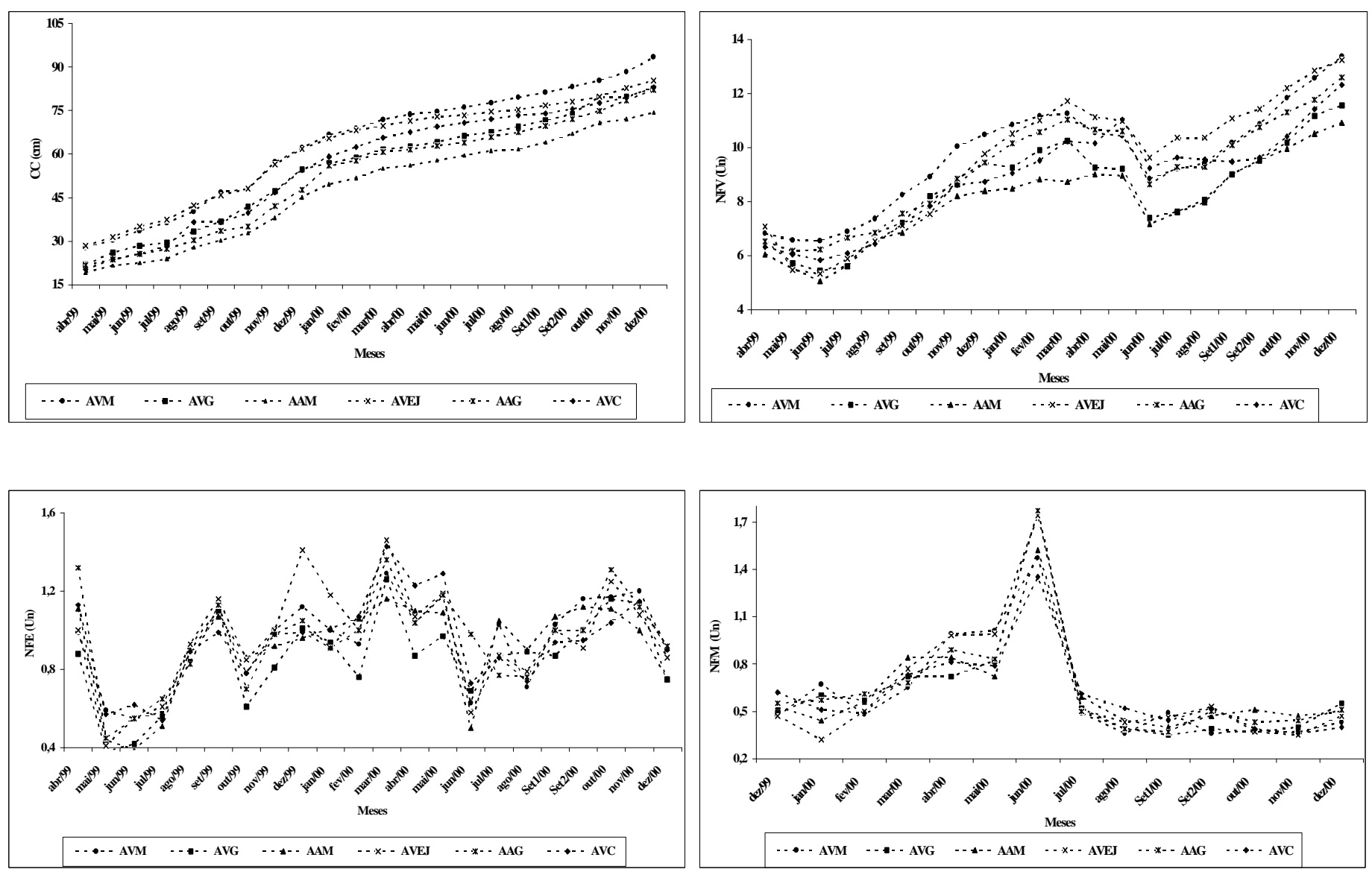

FIGURA 1 - Circunferência do coleto (CC), número de folhas vivas (NFV), número de folhas emitidas (NFE) e número de folhas mortas (NFM), de seis cultivares de coqueiro-anão, em Santo Antônio de Leverger - MT, 2001. 

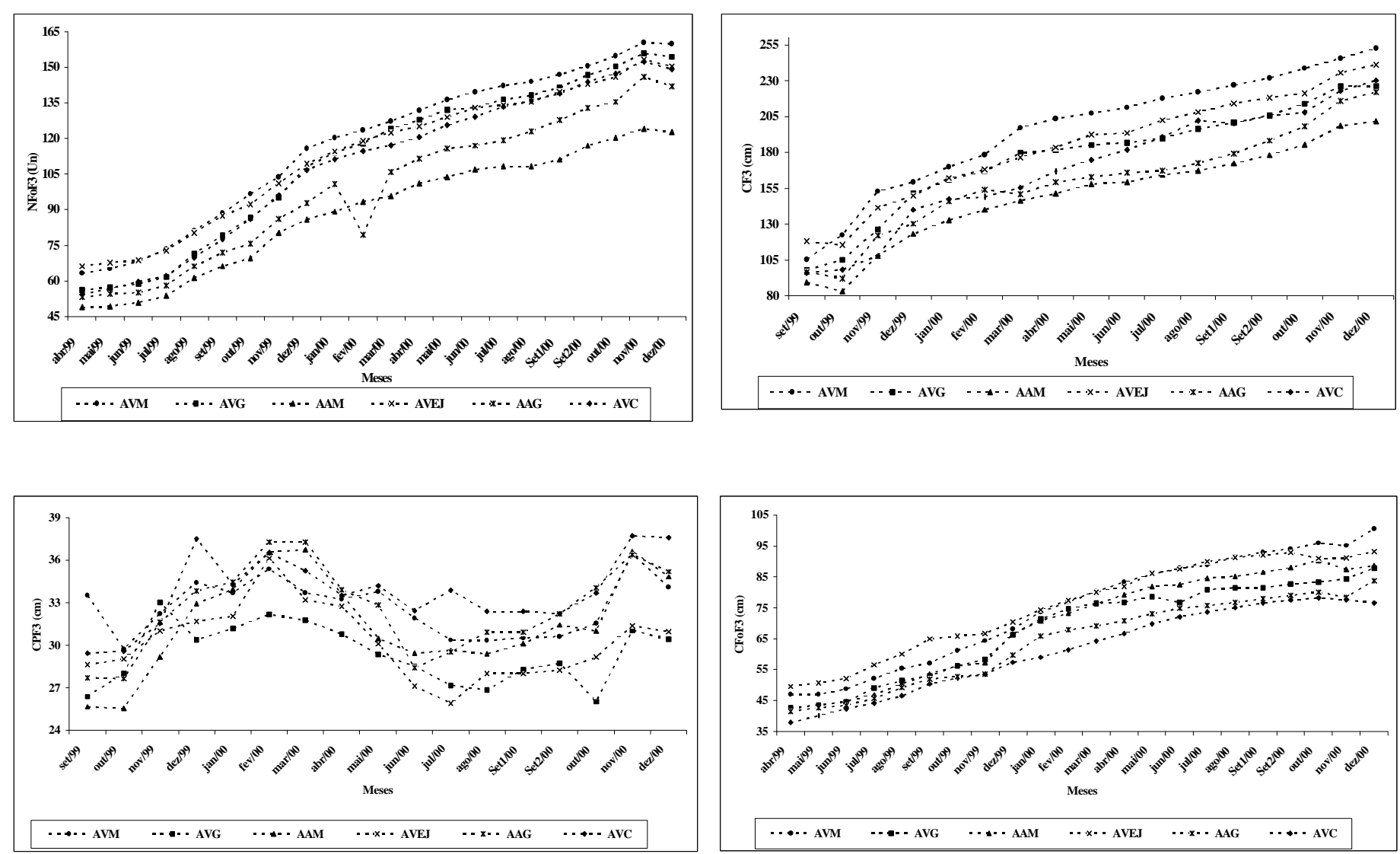

FIGURA 2 - Número de folíolos na folha 3 (NFoF3), comprimento da folha 3 (CF3), comprimento do pecíolo na folha folha 3 (CPF3) e comprimento do folíolo na folha $3(\mathrm{CFoF} 3)$, de seis cultivares de coqueiro-anão, em Santo Antônio de Leverger - MT, 2001.

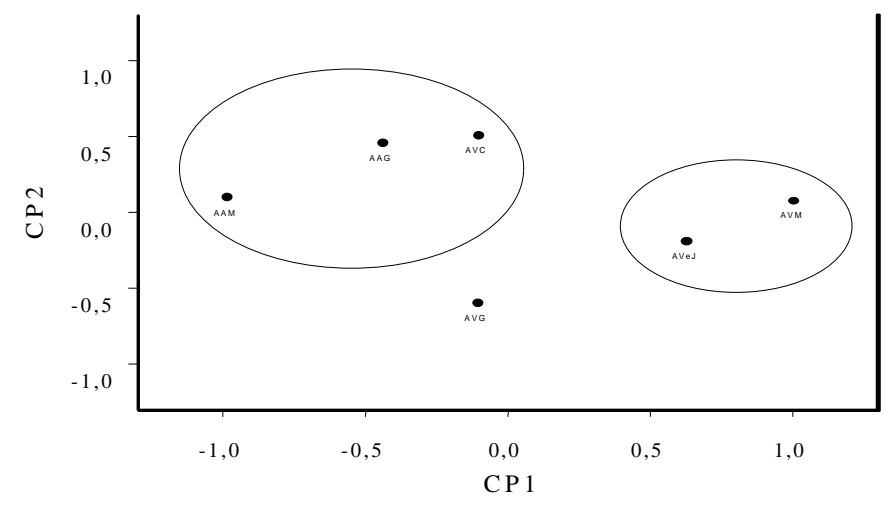

FIGURA 3 - Classificação de seis cultivares de coqueiro-anão, baseada nos escores do primeiro e do segundo componentes principais (CP1 e CP2).
TABELA 1 - Coeficientes da circunferência do coleto (CC), número de folhas vivas (NFV), número de folhas emitidas (NFE), número de folhas mortas (NFM), número de folíolos na folha 3 (NFoF3), comprimento da folha 3 (CF3), comprimento do pecíolo na folha 3 (CPF3) e comprimento do folíolo na folha $3(\mathrm{CFoF} 3)$ no primeiro (CP1) e no segundo $(\mathrm{CP} 2)$ componentes principais

\begin{tabular}{ccc}
\hline Variável & CP1 & CP2 \\
\hline CC & $\mathbf{0 , 2 1 5 7 6}$ & $-0,06017$ \\
NFV & $\mathbf{0 , 1 7 7 7 4}$ & 0,20450 \\
NfoF3 & $\mathbf{0 , 1 9 1 2 7}$ & $-0,13920$ \\
CfoF3 & $\mathbf{0 , 1 7 0 2 9}$ & $-0,19383$ \\
NFE & 0,04410 & $\mathbf{0 , 5 8 9 5 2}$ \\
NFM & $\mathbf{- 0 , 1 6 7 0 8}$ & $-0,04318$ \\
CF3 & $\mathbf{0 , 2 1 5 2 3}$ & $-0,13421$ \\
CPF3 & 0,05283 & $\mathbf{0 , 6 0 4 5 3}$ \\
\hline Variância acumulada & $\mathbf{5 6 , 1 \%}$ & $\mathbf{7 1 , 1 \%}$ \\
\hline
\end{tabular}




\section{CONCLUSÕES}

1. O AAM apresentou menor circunferência do coleto, menor número de folíolos na folha 3 e menor comprimento da folha 3 . 2. Os AVeJ e AVM apresentaram maior crescimento da planta, enquanto o AAM, AAG e AVC apresentaram menor crescimento da planta. O AVG apresentou desempenho intermediário entre os dois grupos.

3. A região de Santo Antônio de Leverger - MT, apresenta condições climáticas adequadas ao crescimento vegetativo das cultivares avaliadas.

\section{REFERÊNCIAS BIBLIOGRÁFICAS}

AKPAN, E. E.J. Evaluation of tall coconut (Cocos nucifera L.) genotypes within the nigerian coconut germplasm bank. Oléagineux, Paris, v.49, n. 1, p.13-20, 1994.

ALDABA, F.R. Coconut production in the Philippines: problems and prospects. Plantations Recherche Developpement, Paris, v. 2, n. 5, p.15-21, 1995.

ARAGÃO, W. M.; CASTILHO, E. L.; FERREIRA, J. M. S.;RIBEIRO, F. E.; TUPINAMBÁ, E. E. M.; FERREIRA, M. de L.; WARWICK, D. R. N. Avaliação de híbridos intervarietais do coqueiro nos tabuleiros costeiros do Sul de Sergipe. Aracaju: EMBRAPA-CPAT, 1997. 3p. (Pesquisa em andamento, 22).

BOURDEIX, R.;CHO, Y. P.; SANGARE, A.; BAUDOIN, L.; DELAMOTHE, M. D.L'hybride de cocotier PB121 amélioré, croisement du Nain Jaune Malais et de géniteurs Grand OuestAfricain selectionnés. Oléagineux, Paris, v.47, n. 11, p.619-633, 1992.

CARADUS, J. R; DUNLOP, J.; WEWALA, S.; MACKAY, A. D.; HART, A. L.; BOSCH, J.; HAY, M. J. M.; LAMBERT, M. G. Nitrogen and phosphorus concentration of white clover genotypes differing in response to added phosphorus. New Zealand Journal of Agricultural Research, Wellington, v. 36, p. 79-86, 1993.

CRUZ, C. D.; REGAZZI, A. J. Modelos biométricos aplicados no melhoramento genético. Viçosa: Imprensa Universitária UFV, 1994.390p.

FERRAZ, L. G. B.; PEDROSA, A. C.; MELO, G. S. de. Avaliação do comportamento de coqueiro híbrido e cultivares nacionais. Recife/PE: IPA, 1987 7p. (Pesquisa em andamento, 5).
FERREIRA, J. M. S.; WARWICK, D. R. N. SIQUEIRA, L.A.(Ed) A cultura do coqueiro no Brasil. Aracaju-SE: EMBRAPA/CPATC, 1998.

IEZZONI, A. F.; PRITTS, M. P. Applications of principal component analysis to horticultural research. HortScience, Alexandria, 26:334-338, 1991.

JOHNSON, R. A.; WICHERN, D. W. Applied multivariate statistical analysis. Upper Saddle River: Prentice Hall, 1998. 816 p.

NUCE de LAMOTHE, M. de; SANGARE, A.; MEUNIER, J.; LE SAINT, J. P. Coconut hybrids - interest and prospects, IRHO contribution to research and development. In: SILAS, E. G.; ARAVINDAKSHAN, M.; JOSE, A. I. Coconut breeding and management. Vellinakkara, Karala Agricultural University, 1991. p.26-38.

PASSOS, E. E. M. Morfologia do coqueiro. In: FERREIRA, J. M. S.; WARWICK, D. R. N.; SIQUEIRA, L.A.(Ed), A cultura do coqueiro no Brasil. Aracaju-SE: EMBRAPA/CPATC, 1998. p. 57-64.

PERSLEY, G. J. Replanting the tree of life: Towards an international agend for coconut palm research. Wallingford: CAB, 1992. 133p.

RIBEIRO, F. E.; SIQUEIRA, E. R. de; ARAGÃO, W. M. de; TUPINAMBÁ, E. A. O coqueiro-anão no Brasil. Aracaju: Embrapa-CPATC, 1999. 22p. (Documentos, 8).

SAABOR, A.; LOPES, L. H. S.; CUNHA, M. M.; FERNANDES, C. - Coco verde: Ministério da Integração Nacional - MI., 2000. (Frutiséries, 3).

SANTOS, G. A.; CARPIO, C. B.; ILAGAN, M. C.; CANO, S. B.; DELA CRUZ, B. V. - Flowering and early yield performance of four IRHO coconuts hybrids in the Philippines. Oléagineux, Paris, v. 37, n.12, p.571-580, 1982.

STEWART, D. P. C.; CAMERON, K. C.; CORNFORTH, I. S.; SEDCOLE, J. R. Effects of spent mushroom substrate on soil physical conditions and plant growth in an intensive horticultural system. Australian Journal of Soil Research, 36: 899-912, 1998.

ZUSHUN, M. A new coconut hybrid, WY78F1. Oléagineux, Paris, v.49, n. 2, p.49-54, 1994. 